\title{
THE COLD GAS CONTENT OF ELLIPTICAL GALAXIES
}

\author{
W.K. HUCHTMEIER \\ Max-Planck-Institut für Radioastronomie \\ Auf dem Hügel 69, D-53121 Bonn, Germany \\ L.J. SAGE \\ Department of Astronomy, University of Maryland \\ College Park MD 20742, USA \\ AND \\ C. HENKEL \\ Max-Planck-Institut für Radioastronomie \\ Auf dem Hügel 69, D-53121 Bonn, Germany
}

The $100 \mathrm{~m}$ radiotelescope at Effelsberg has been used to observe two samples of elliptical galaxies in the $21 \mathrm{~cm}$ line of neutral hydrogen. One sample is defined by the elliptical galaxies in the Revised-Shapely-Ames catalog (RSA) (Huchtmeier 1994, Astron.Astrophys286, p.389); the other sample is defined by all elliptical galaxies with IRAS $100 \mu$ fluxes $\geq 500 \mathrm{mJy}$ north of declination $-31^{0}$ (Huchtmeier, Sage, Henkel 1995 Astron.Astrophys. in press). Among the detected galaxies there are 23 (RSA) and 24 (IRAS) isolated elliptical galaxies free of confusion by nearby galaxies with similar radial velocities. Global properties of these two samples of elliptical galaxies are discussed: their HI-properties, optical and IR luminosities, their optical colors, their masses of dust and of molecular hydrogen.

Elliptical galaxies from the RSA and most elliptical galaxies from the IRAS sample have the same mean $M_{H I} / L_{B}$ ratio : $0.030 \pm 0.026$; only a small group of objects from the IRAS sample is several times richer in HI $\left(M_{H I} / L_{B}=0.206 \pm 0.105\right)$. These "HI-rich" elliptical galaxies have blue colors like spiral galaxies and have a tendency towards higher average dust temperatures. The large number of elliptical galaxies in compact groups (in this sample) suggests that gravitational interactions and mergers may be an important source of interstellar matter for elliptical galaxies. 